\title{
Un déficit immunitaire combiné est lié à des mutations dans le gène de la tyrosine kinase ZAP-70
}

Les lymphocytes $\mathrm{T}$ et $\mathrm{B}$ portent à leur surface des récepteurs qui reconnaissent spécifiquement les antigènes. Ces lymphocytes sont habituellement au repos mais peuvent être activés lors du contact du récepteur de l'antigène (TcR) avec son ligand (Ag) qui entraîne la transmission de signaux intracellulaires et déclenche une réponse immunitaire spécifique. Les récepteurs des antigènes sont suppléés par des corécepteurs (CD4 et CD8 pour les lymphocytes T) qui participent directement à la formation des complexes Ag-TcR et contribuent à l'initiation des signaux d'activation. D'autres molécules contribuent à l'activation cellulaire en permettant la transmission de signaux additionnels (système CD28-B7) et en augmentant l'avidité de l'interaction AgTcR (molécules d'adhérence). Au cours de l'ontogénie des lymphocytes, les thymocytes corticaux réarrangent leurs gènes $\alpha$ et $\beta$ du TcR et expriment simultanément les corécepteurs CD4 et CD8 au stade dit " double positif". Les thymocytes médullaires sont plus différenciés et n'expriment que l'un des deux corécepteurs (stade "simple positif" $\mathrm{CD}^{+}$ou $\left.\mathrm{CD}^{+}\right)$. Cette transition dépend de l'interaction entre le TcR et le complexe majeur d'histocompatibilité $(\mathrm{CMH})$ qui déclenche le processus de sélection positive des thymocytes. La sélection positive des thymocytes $\mathrm{CD}^{+}$passe par la reconnaissance du CMH-I alors que les thymocytes $\mathrm{CD}^{+}$sont sélectionnés sur leur capacité à reconnaître le CMH-II.

Les récepteurs des antigènes n'ont 1038

phosphatase intrinsèque mais activent des protéine-tyrosine kinases (PTK) cytoplasmiques appartenant aux familles Src (Lck et Fyn) et Syk/ZAP-70 [1]. En aval des PTK, la stimulation des lymphocytes $T$ passe par la voie du phosphatidylinositol, l'activation de Ras et de sérine/thréonine kinases et phosphatases. Cette cascade d'événements conduit à des réponses cellulaires très diversifiées qui dépendent de la cellule, de son état de différenciation et du contexte dans lequel se fait la reconnaissance de l'antigène. Ainsi, l'interaction du récepteur de l'antigène avec son ligand peut conduire à l'apoptose, l'anergie ou la prolifération du lymphocyte, la frontière entre les trois états n'étant pas clairement définie. La transmission de signaux par le TcR est donc un événement crucial de la différenciation intrathymique et de l'activation des lymphocytes $\mathrm{T}$, mais la participation respective des PTK à cette fonction du TcR n'a pas été établie définitivement. Fyn est impliquée dans la transmission des signaux dans les thymocytes "simple positif " et au moment de la sélection négative des cellules autoréactives [2,3]. Lck semble associée directement à CD4 et CD8 et être indispensable à une activation cellulaire complète faisant suite à une stimulation antigénique $[4,5]$. L'absence de Lck se traduit par une réduction du nombre de thymocytes et de cellules $\mathrm{T}$ périphériques [6]. Lck interviendrait aussi très précocement au cours de la différenciation thymique, à un stade où ni le TcR ni les corécepteurs ne sont exprimés. Au contraire des PTK de la famille Src, Syk et ZAP-70 ne sont pas myristoylées et ne semblent pas constitutivement associées à la membrane plasmique. Les membres de la famille Syk/ZAP70 ont deux domaines SH2 aminoterminaux et un domaine C-terminal catalytique. ZAP-70 est exclusivement exprimée par les lymphocytes $\mathrm{T}$ et les cellules tueuses NK (natural killer) alors que Syk est exprimée préférentiellement par les lymphocytes B, les cellules myéloïdes et les thymocytes [1].

Les récepteurs des antigènes des lymphocytes $\mathrm{T}$ et $\mathrm{B}$ sont constitués de deux modules associés de façon non covalente. L'un contient les sous-unités liant l'antigène et l'autre regroupe les sous-unités spécialisées dans la transmission des signaux. Ces dernières possèdent des motifs communs cytoplasmiques (séquence ARAM : antigen recognition activation motif) permettant leur couplage aux PTK intracellulaires [7]. A la faveur de la reconnaissance de l'Ag, le récepteur interagirait séquentiellement avec les deux types de PTK. Un membre de la famille Src phosphorylerait des motifs ARAM de certaines sous-unités du TcR qui pourraient alors s'associer avec les domaines SH2 de ZAP-70. ZAP-70 aurait donc un rôle plus tardif dans la transmission des signaux et sa fonction serait partiellement contrôlée par les tyrosine kinases Src.

Le rôle de ZAP-70 a pu être évalué grâce à l'étude moléculaire de quelques cas de déficit immunitaire combiné chez l'homme [8-10]. Les déficits immunitaires combinés constituent un groupe hétérogène de maladies génétiques pour lesquelles le défaut n'est pas toujours connu. Chez certains malades, les 
lymphocytes circulants expriment tous le corécepteur CD4 et aucune molécule CD8 ne peut être détectée à leur surface. Le nombre total de lymphocytes et la proportion des lymphocytes $\mathrm{T}$ sont pourtant respectés. Cette anomalie semble liée à un défaut dans la sélection positive des thymocytes $\mathrm{CD}^{+}$, car les thymocytes corticaux "double positifs" existent alors que seule la population "simple positive" $\mathrm{CD}^{+}$est détectable dans la medulla thymique. Les lymphocytes $\mathrm{CD}^{+}$des patients sont d'origine polyclonale, expriment d'autres marqueurs de différenciation lymphocytaire mais ne prolifèrent pas en réponse aux mitogènes ou aux anticorps monoclonaux dirigés contre le TcR lui-même. En revanche, ils prolifèrent en présence d'esters de phorbol et d'un ionophore de calcium, une stimulation qui n'emprunte pas la voie précoce de signalisation par le TcR. Une anomalie du couplage du TcR avec les PTK cytoplasmiques a donc été suspectée pour ce type d'immunodéficience.

Trois des patients décrits sont homozygotes pour une mutation ponctuelle dans un intron du gène ZAP-70. Cette mutation se traduit par un épissage anormal et une insertion de trois acides aminés dans le domaine kinase de la protéine, entraînant la production d'une protéine instable, sans activité kinasique. Dans une autre étude, une mutation semblable a été identifiée dans l'allèle maternel de trois enfants malades d'une même famille. Chez ces enfants, l'allèle paternel contient aussi une mutation ponctuelle qui provoque le remplacement, dans le domaine catalytique de la protéine, d'une sérine très conservée par une arginine. Chez une autre malade, une délétion de treize paires de bases entraîne un changement dans le cadre de lecture et la synthèse d'une protéine écourtée de 82 acides aminés. Cette malade est homozygote pour cette mutation.

L'étude des lymphocytes $\mathrm{CD}^{+}{ }^{+}$périphériques des malades montre que les phosphorylations des tyrosines et l'activation de la phospholipase-C $\gamma$ induites par la stimulation du TcR sont partiellement sous le contrôle de ZAP-70, alors que les événements plus tardifs de l'activation cellulaire, comme la production d'IL2 et la prolifération, sont complètement dépendants de ZAP-70.

I.es voies de signalisation du TcR varient selon le stade de différenciation des lymphocytes et ZAP-70 semble donc être indispensable au développement des lymphocytes $\mathrm{CD}^{+}$et au bon fonctionnement des lymphocytes $\mathrm{CD} 4^{+}$. En revanche, ZAP-70 ne jouerait pas un rôle essentiel dans la sélection thymique des thymocytes $\mathrm{CD} 4^{+} \mathrm{CD} 8$.

V.L.

1. W'eiss $\lambda$, I.ittman DR. Signal transduction by lymphocyte antigen receptors. Ciell 1994; 76 : 263-74.

2. Stern PL, I.ee HM, Rich S, Sorino P. pp59fyn mulant mice display differential signaling in thymocytes and peripheral $\mathrm{T}$ cells. Cell $1992 ; 70$ : 74 1-50.

3. Appleby MW', Gross JA, Cook MP, et al. Defective $T$ cell receptor signaling in mice lacking the thymic isof orm of p59fyn. Cell 1992; 70:751-63. 4. Veillette A, Zuinuga-Pflucker IJ, Bolen JB, Kuisbeeck AM. Engagement of (D) and (:D) 8 expressed on immature thymocytes induced activation of intracellular tyrosine phosphorylation pathways. JExp Med 1989 ; 170 : 1671-89.

5. Glaichen ha us $N$, Shastri N, I.itman D, Turner J. Requirement for association of $p 56^{\text {lsk }}$ with (D4 antigen specific signal transduction in T cells. Cell 1991; 64: 511-20.

6. Molina TJ, Kishihara K. Siderouski DP, et al. Profound block in thymocyte development in mice lacking $\mathrm{p} 56^{\text {th }}$. Nature $1992 ; 357$ : 161-4.

7. W'eiss A. T cell antigen receptor signal transduction : a tale of tails and cytoplasnic proteintyrosine kinases. Cell 1993; 73: 209-12.

8. Arpaia E, Shahar M, Dadi H, Cohen A, Roifman C.M. Defective T cell receptor signaling and (.) $8^{+}$thymic selection in humans lacking ZAP-70 kinase. Cell $1994 ; 76$ : 947-58.

9. Elder ME, Lin D, Clever J, Hope TJ, W'eiss A, Parslow TG. Human severe combined immunodeficiency due to a deficit in ZAP-70, a T cell tyrosine kinase. Science $1994 ; 264: 1596-9$

10. Chan AC, Kadlecek TA, Elder ME, et al. ZAP70 deficiency in an autosomal recessive form of severe combined immunodeficiency. Science $1994 ; 264: 1599-1601$.
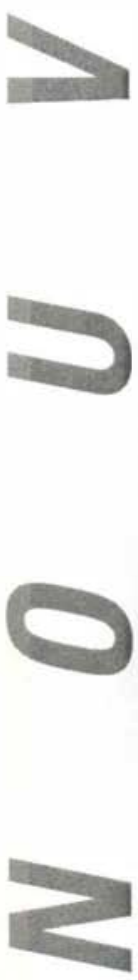